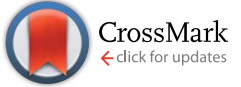

Cite this: RSC Adv., 2017, 7, 11636

Received 30th December 2016 Accepted 28th January 2017

DOI: $10.1039 / c 6 r a 28838 c$

rsc.li/rsc-advances

\section{Films based on group IV-V-VI elements for the design of a large-gap quantum spin Hall insulator with tunable Rashba splitting}

\author{
Yi-zhen Jia, Wei-xiao Ji, Chang-wen Zhang, * Shu-feng Zhang, Ping Li \\ and Pei-ji Wang
}

\begin{abstract}
Rashba spin-orbit coupling (SOC) in topological insulators (TIS) has recently attracted significant interest due to its potential applications in spintronics. However, to date, the coexistence of a giant Rashba SOC and band topology has rarely been investigated in two-dimensional (2D) films. Herein, we applied firstprinciples calculations to design a family of large-gap 2D topological insulators composed of hexagonal $\mathrm{Bi}$ and $\mathrm{PbX}(\mathrm{X}=\mathrm{F}, \mathrm{Cl}, \mathrm{Br}$, and $\mathrm{I})$ dimers. The nontrivial topology, induced via a $\mathrm{p}_{x y}-\mathrm{p}_{z}$ band inversion, was confirmed by the $Z_{2}$ index and helical edge states. Note that the Rashba splitting energy in these films reaches $81 \mathrm{meV}$, which is further tunable over a wide range of strains $(-2-14 \%)$. Considering the robustness of the band topology on a h-BN substrate, this study provides a route for designing topological spintronic devices based on 2D films consisting of group IV-V-VI elements.
\end{abstract}

Spin-splitting plays a key role in spintronic devices due to spinpolarized currents, spin injection, and spin accumulation., ${ }^{1,2}$ The main feature of the Rashba effect, i.e., spin splitting in twodimensional (2D) electronic states from the perpendicular potential asymmetry with spin-orbit coupling (SOC), lies in the possibility of controlling the spin states in the absence of a magnetic field., ${ }^{3,4}$ Typical Rashba effects have been previously reported in many systems. However, some semiconductor heterostructures ${ }^{5}$ exhibit a small Rashba splitting, with the strength determined by a coefficient $\alpha_{\mathrm{R}}$ of $\sim 0.07 \mathrm{eV} \AA$; moreover, noble metal ${ }^{6,7}$ and heavy metal surfaces ${ }^{8,9}$ exhibit larger Rashba splitting, with $\alpha_{\mathrm{R}}$ in the range of $0.33-1.30 \mathrm{eV} \AA$. Interestingly, the Rashba splitting can be further enhanced in several systems, such as a Bi/Ag alloy, ${ }^{10} \mathrm{Bi}$ quantum film on $\mathrm{Cu}(111),{ }^{11}$ and the bulk polar BiTeI (1.36-5 eVÅ). ${ }^{12}$ Recently, Rashba spin splitting has also been observed for molecule adsorption on ferroelectric GeTe, ${ }^{13} \mathrm{CH}_{3} \mathrm{NH}_{3} \mathrm{PbX}_{3}$ hybrid organic-inorganic halide perovskites, ${ }^{14}$ and CdTe/InSb heterostructures. ${ }^{15}$ These results pave the way for the design of novel spin field-effect transistors.

In addition to the Rashba splitting, another prominent feature induced by SOC is the topological nontrivial band structure..$^{16,17}$ These novel materials are insulating in the bulk, whereas exhibit gapless surface (edge) states protected by the time-reversal invariance. ${ }^{18}$ As compared with experimentally synthesized 3D topological insulators (TIs), ${ }^{19-22}$ 2D TIs are better suited for applications in quantum computing due to the remarkable robustness of edge states against backscattering. The current

School of Physics and Technology, University of Jinan, Jinan, Shandong, 250022, People's Republic of China. E-mail: ss_zhangchw@ujn.edu.cn experimental realization of 2D quantum spin-Hall (QSH) phases, however, is only limited to $\mathrm{HgTe} / \mathrm{CdTe}^{23,24}$ and InAs/GaSb ${ }^{25,26}$ quantum wells at ultra-low temperatures $(<10 \mathrm{~K})$. Subsequently, several large-gap 2D QSH phases, such as germanium, ${ }^{27}$ tin, ${ }^{28}$ arsenene, ${ }^{29} \mathrm{Bi}(111),{ }^{30} \mathrm{III}-\mathrm{Bi},{ }^{31} \mathrm{BiF},{ }^{32} \mathrm{Bi}_{4} \mathrm{Br}_{4},{ }^{33} \mathrm{GaAs},{ }^{34}$ as well as functionalized films have been reported in theoretical studies. ${ }^{34-45}$ As compared with centrosymmetric TIs, non-centrosymmetric topological phases are more promising due to their perfect performance in realizing novel quantum phenomena, such as crystalline-surface dependent topological states, pyroelectricity, ${ }^{46}$ natural topological p-n junctions ${ }^{47}$ and so on. However, to the best of our knowledge, the coexistence of a large Rashba splitting with topological properties, which is the focus of this study, has rarely been reported in 2D materials.

Among group-V elements, Bi has the largest SOC strength, and thus, many Bi-based compounds have been designed to realize large-gap topological phases. ${ }^{29-32}$ On the other hand, lead $(\mathrm{Pb})$, the Bi counterpart in the group IV, with the largest SOC among group-IV elements, is also a promising candidate for realizing large-gap 2D TIs. ${ }^{40}$ These considerations prompted us to wonder whether a new $2 \mathrm{D}$ film composed of both $\mathrm{Bi}$ and $\mathrm{Pb}$ atoms could be constructed and whether this film could exhibit a larger Rashba splitting due to inversion-symmetry breaking.

In the present study, we designed a family of $2 \mathrm{D}$ hexagonal films with $\mathrm{Bi}$ and $\mathrm{PbX}$ dimers, $\mathrm{BiPbX}(\mathrm{X}=\mathrm{F}, \mathrm{Cl}, \mathrm{Br}$, and $\mathrm{I})$, whose structural stability was confirmed by phonon spectrum calculations. These BiPbX films were proven to be 2D TIs with bulk band gaps as large as $0.28-0.32 \mathrm{eV}$. Their nontrivial band topology is attributed to a $\mathrm{p}_{z}-\mathrm{p}_{x y}$ band inversion, which is evidenced by the helical edge states. Especially, a giant Rashba splitting can be 
obtained, due to the polar atomic layers of $\mathrm{Bi}$ and $\mathrm{PbX}$ dimers. The topological order is robust against external strain, whereas interestingly, the Rashba splitting energy can reach a maximum of $81 \mathrm{meV}$ over a wide range of strains $(-2-14 \%)$. Moreover, we found that these results remained unchanged on a h-BN substrate. Thus, our proposed new structures, with both a nontrivial band structure and giant Rashba splitting, present a promising platform in spintronics and quantum computational applications.

All the calculations were performed using the plane wave basis Vienna ab initio simulation package (VASP) code $^{48,49}$ based on density functional theory (DFT). The projector-augmented wave (PAW) method $^{50}$ was used to describe the electron-ion potential, and the exchange-correlation potential was approximated by the generalized gradient approximation (GGA) in the Perdew-Burke-Ernzerhof (PBE) $)^{51}$ form of the exchangecorrelation function. Due to the underestimation of the band gap, the Heyd-Scuseria-Ernzerhof (HSE06) hybrid functional ${ }^{52,53}$ was employed to check the band structures. We applied an energy cut-off of $500 \mathrm{eV}$ and a maximum residual force of less than $0.001 \mathrm{eV}^{-1}$. To simulate the $2 \mathrm{D}$ crystals, periodic boundary conditions were applied, and the Brillouin zone was sampled using a $15 \times 15 \times 1$ Gamma-centered Monkhorst-Pack grid. Moreover, SOC effects were included in the self-consistent electronic structure calculations. The phonon spectra were obtained by the supercell approach within the PHONON code.

Fig. 1 shows the geometrical structure of BiPbX films, with $\mathrm{Bi}$ and $\mathrm{Pb} \mathrm{sp}^{3}$ orbitals strongly hybridized, in a way akin to semihydrogenated silicene ${ }^{54,55} \quad\left(C_{3 \mathrm{v}}\right.$ symmetry $)$. The structural stability of the films was evaluated by the formation energy, defined as

$$
\Delta E_{\mathrm{f}}=E(\mathrm{BiPbX})-E(\mathrm{BiPb})-E\left(\mathrm{X}_{2}\right) / 2
$$

where $E(\mathrm{BiPbX})$ and $E(\mathrm{BiPb})$ are the total energies of halogenated and pristine BiPb monolayers, respectively, whereas $E\left(\mathrm{X}_{2}\right)$ is the chemical potential of halogen atoms. Table 1 lists the calculated $\Delta E_{\mathrm{f}}$, equilibrium lattice constants, buckling heights, as well as the $\mathrm{Bi}-\mathrm{Pb}$ and $\mathrm{Pb}-\mathrm{X}$ bond lengths after structural optimization. The results for the $\Delta E_{\mathrm{f}}$ of BiPbF, BiPbCl, BiPbBr, and BiPbI are $-2.64,-1.28,-1.13$, and $-0.88 \mathrm{eV}$, respectively, which demonstrate a higher stability for phases containing halogens with higher electronegativity. Furthermore, we calculated the phonon dispersion spectra of all these $2 \mathrm{D}$ films. As illustrated in Fig. 1(c), they are dynamically stable because all the vibrational modes are real in the whole Brillouin zone.

To test the thermostability of the BiPbX monolayers, we performed first-principles molecular dynamics (MD) simulations. We used a $4 \times 4$ supercell and carried out the individual $\mathrm{MD}$ simulations for $\mathrm{BiPbBr}$ as an example. As can be seen from Fig. 2, the structure of the $\mathrm{BiPbBr}$ monolayer does not collapse throughout a 5 ps MD simulation up to $300 \mathrm{~K}$, indicating that the melting point of the BiPbBr monolayer is probably $300 \mathrm{~K}$. The abovementioned results reveal that the $\mathrm{BiPbBr}$ monolayer has a very good thermal stability and can maintain its structural integrity at high temperatures.

The calculated band structures for $\mathrm{BiPbX}$ with $\mathrm{X}=(\mathrm{F}, \mathrm{Cl}, \mathrm{Br}$, and I) are shown in Fig. 3, where the red and blue dots represent the $\mathrm{p}_{z}$ and $\mathrm{p}_{x, y}$ states, respectively. Without including SOC effects, they exhibit semiconducting character, with the valence band maximum (VBM) and the conduction band minimum (CBM) degenerating at the $I$ point. Upon increasing the atomic (a)
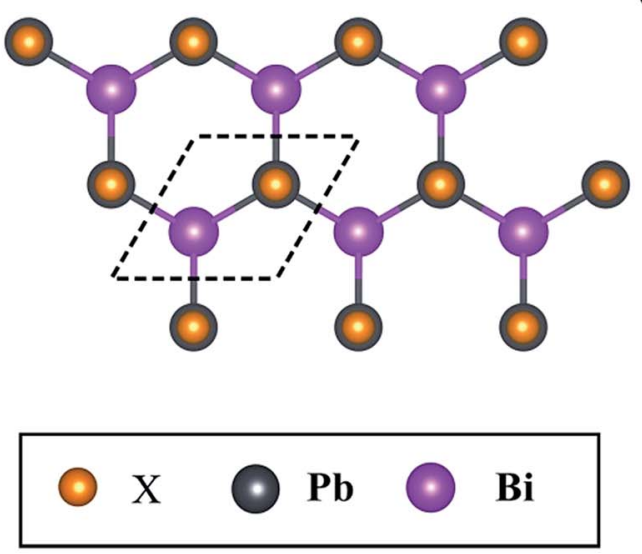

(b)

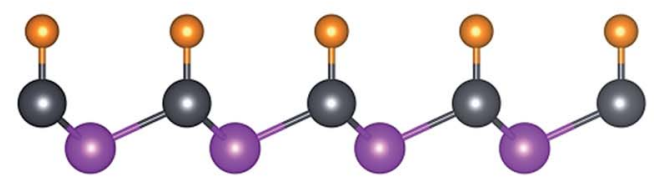

(c)
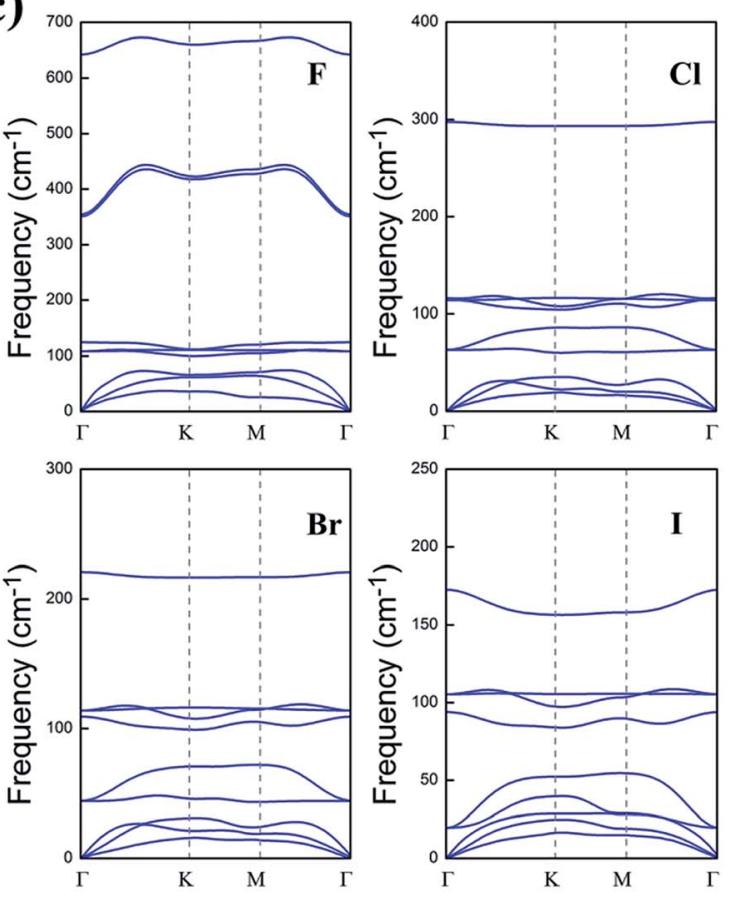

Fig. 1 (a) Top view and (b) side view of the $\mathrm{BiPbX}(\mathrm{X}=\mathrm{F}, \mathrm{Cl}, \mathrm{Br}$, and I) monolayer, and (c) phonon band dispersions of BiPbX. 
Table 1 Calculated equilibrium lattice parameters a $(\AA)$, buckled height $h(\AA)$, Bi-Pb bond length $d_{\mathrm{Bi}-\mathrm{Pb}}(\AA)$, $\mathrm{Pb}-\mathrm{X}$ bond length $d_{\mathrm{Pb}-\mathrm{X}}(\AA)$, formation energy $\Delta E_{\mathrm{f}}(\mathrm{eV})$, band gap without SOC effects $E_{\mathrm{g}}(\mathrm{eV})$, band gap with SOC effects $E_{\mathrm{g}-\mathrm{SOC}}(\mathrm{eV})$, band gap at the $\Gamma$ point without SOC effects $E_{\Gamma}(\mathrm{eV})$, band gap at the $I$ point with SOC effects $E_{T-\text { sOc }}(\mathrm{eV})$, and topological invariants $\left(Z_{2}\right)$ for $\mathrm{BiPbX}$

\begin{tabular}{|c|c|c|c|c|c|c|c|c|c|c|}
\hline Structure & $a(\AA)$ & $h(\AA)$ & $d_{\mathrm{Bi}-\mathrm{Pb}}(\AA)$ & $d_{\mathrm{Pb}-\mathrm{X}}(\AA)$ & $\Delta E_{\mathrm{f}}(\mathrm{eV})$ & $E_{\mathrm{g}}(\mathrm{eV})$ & $E_{\Gamma}(\mathrm{eV})$ & $E_{\mathrm{g}-\mathrm{SOC}}(\mathrm{eV})$ & $E_{\Gamma \text {-SOC }}(\mathrm{eV})$ & $Z_{2}$ \\
\hline $\mathrm{BiPbF}$ & 4.78 & 1.28 & 3.04 & 2.06 & -2.64 & 0.217 & 0.217 & 0.232 & 0.278 & 1 \\
\hline $\mathrm{BiPbCl}$ & 4.75 & 1.32 & 3.04 & 2.46 & -1.28 & 0.195 & 0.195 & 0.229 & 0.275 & 1 \\
\hline $\mathrm{BiPbBr}$ & 4.73 & 1.34 & 3.04 & 2.60 & -1.13 & 0.163 & 0.163 & 0.196 & 0.288 & 1 \\
\hline $\mathrm{BiPbI}$ & 4.72 & 1.35 & 3.04 & 2.81 & -0.88 & 0.071 & 0.071 & 0.138 & 0.320 & 1 \\
\hline
\end{tabular}

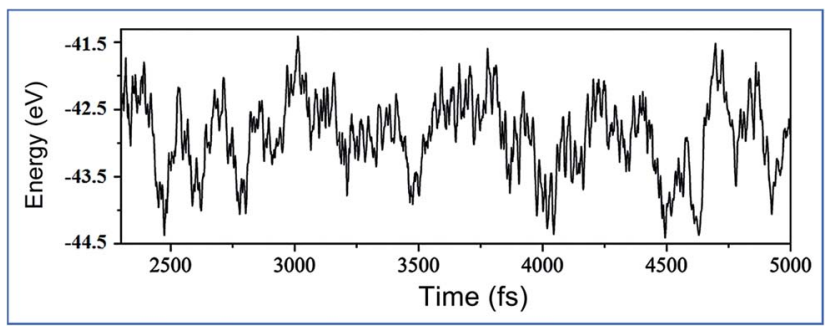

Fig. 2 Total potential energy fluctuations during the MD simulations at $300 \mathrm{~K}$ for the $\mathrm{BiPbBr}$ monolayer.

number of the halogen in the $\mathrm{PbX}$ dimer, the band gap continuously decreases, reaching $71 \mathrm{meV}$ for the PbI dimer. When including SOC effects, the CBM, which is mainly occupied by $\mathrm{p}_{z}$ orbitals, is pushed downwards, whereas the VBM, which is occupied by $\mathrm{p}_{x, y}$ orbitals, is reversely pushed upwards. Due to the strong SOC effects of the $\mathrm{Bi}$ and $\mathrm{Pb}$ atoms, the band order around the $\Gamma$ point is inverted, leading to the appearance of a Mexican hat shape near the VBM, which is opposite to the band state alignment without including the SOC effects. This $\mathrm{p}_{x, y}-\mathrm{p}_{z}$ band inversion strongly indicates the topologically nontrivial nature of these films. To overcome the possible underestimation of the band gap, we also employed the HSE06 functional to check the existence of band inversion, as shown in Fig. 4. Note that the nontrivial band gap is enlarged to 0.53$0.72 \mathrm{eV}$, which is larger than that of $\mathrm{Bi}(111)(0.2 \mathrm{eV}),{ }^{14} \mathrm{ZrTe}_{5}$ $(0.1 \mathrm{eV}),{ }^{56}$ as well as stanene $(0.1 \mathrm{eV}) .{ }^{13}$ The large nontrivial band gap of these monolayers is very favourable for future experimental applications and makes them highly adaptable to various application fields.

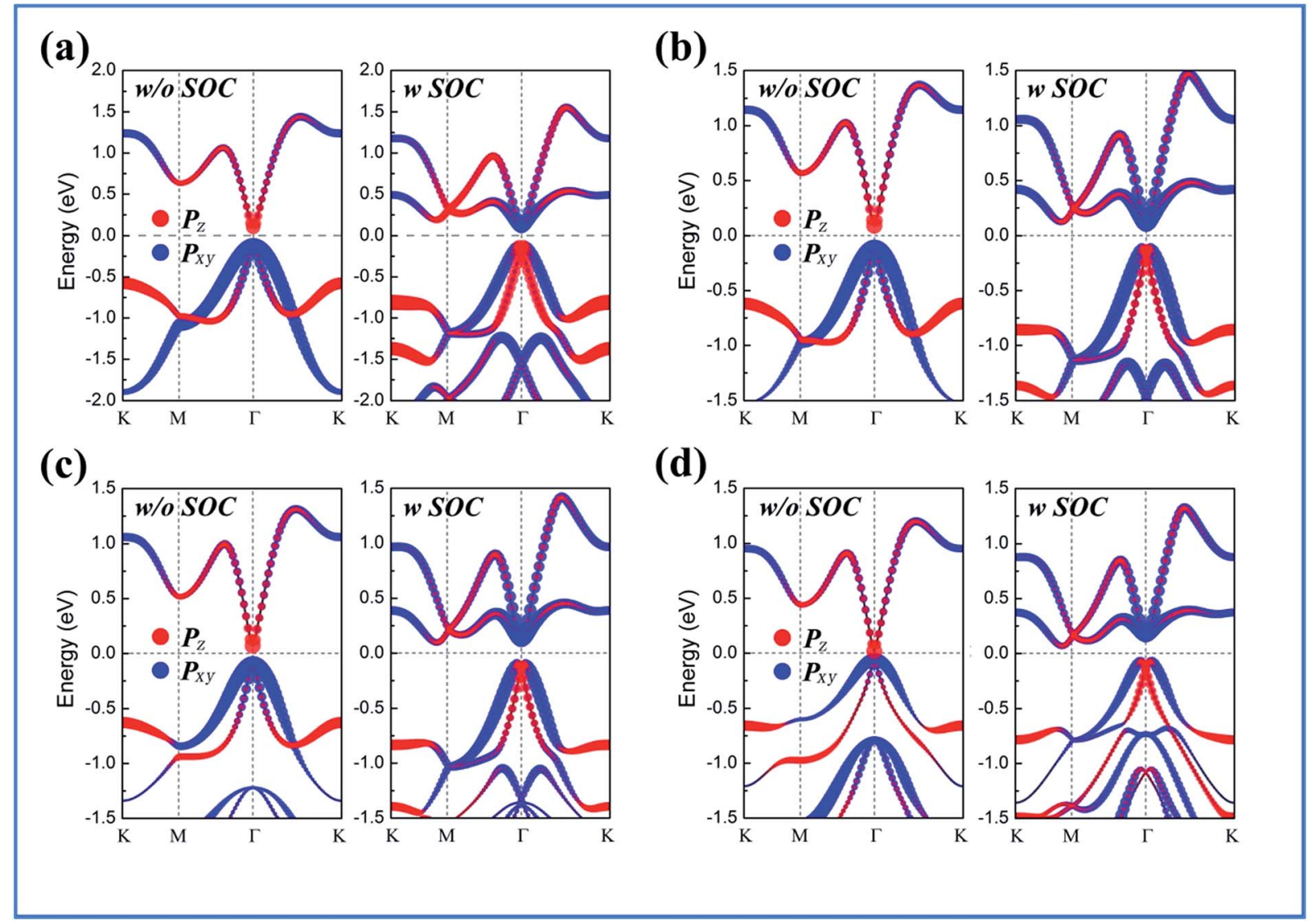

Fig. 3 Orbital-resolved band structures of (a) BiPbF, (b) BiPbCl, (c) BiPbBr, and (d) BiPbl, without $\mathrm{SOC}$ and with $\mathrm{SOC}$ effects. 


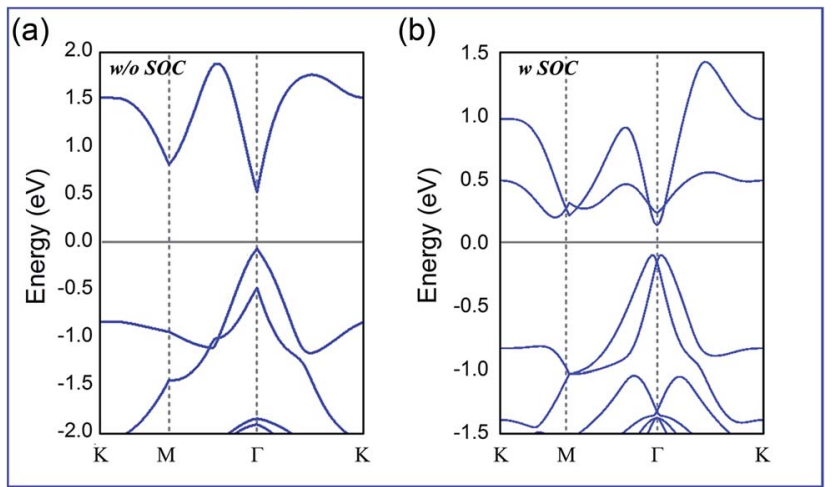

Fig. 4 Calculated band structures of the $\mathrm{BiPbBr}$ monolayer by the HSE06 functional (a) without SOC and (b) with SOC effects.

The hallmark of the QSH effect is the helical edge state, which is the key to spintronics due to their ability to conduct dissipationless currents. To explicitly see these topological features, the edge Green's functions were calculated by the $a b$ initio tight-binding (TB) model, with the recursive method in terms of maximally localized Wannier functions (MLWFs). ${ }^{57}$ As the energy bands near the Fermi level are predominantly composed of hybridized $\mathrm{p}_{z}$ and $\mathrm{p}_{x, y}$ orbitals, the MLWFs are mainly composed of p-like atomic orbitals. Fig. 5(b)and (c) display the bands fitted by the DFT and TB model, as well as the local density of states (LDOS) on the edge states. The good fitting confirms the validity of our results. Obviously, all the edge bands completely connect the conduction and valence bands and span the 2D bulk band gap, yielding $1 \mathrm{D}$ gapless edge states. Furthermore, the band topology was evaluated by the $Z_{2}$ topological invariant. Due to the inversion-symmetry breaking, the $Z_{2}$ invariant can be obtained to trace the Wannier Center of Charges (WCCs) using a non-Abelian Berry connection. ${ }^{58}$ By counting the number of crossings of any arbitrary horizontal reference line, it was found that the WCCs evolution curves cross any arbitrary reference lines in odd number of times, yielding $Z_{2}=1$, as shown in Fig. 5(a).

Then, we focused on the origin of the QSH effect in these systems. As abovementioned, the hybridization of halogen atoms with $\mathrm{Pb}-\mathrm{p}_{z}$ orbital occurs in the $\mathrm{PbX}$ dimers, the $\mathrm{p}_{z}$ band is shifted away from the Fermi level, leaving only $\mathrm{Pb}-\mathrm{p}_{x, y}$ orbitals and $\mathrm{Bi}-\mathrm{p}_{z}$ orbitals, as displayed in Fig. 3. Thus, the evolution of the atomic orbitals can be summarized in three stages: (I), (II), and (III), as shown in Fig. 6(a). In stage (I), the crystal field makes the $\mathrm{p}$ states split into both $\mathrm{p}_{x, y}$ and $\mathrm{p}_{z}$ orbitals due to $C_{3}$ symmetry, with $\mathrm{p}_{z}$ shifting upwards with respect to the degenerate $\mathrm{p}_{x, y}$ orbitals. When chemical bonding occurs between $\mathrm{Bi}$ and $\mathrm{PbX}$ dimer, in stage (II), the states near the Fermi level are further split into bonding and anti-bonding states, i.e., $\mathrm{p}_{x, y}{ }^{+}$, $\mathrm{p}_{x, y, z}{ }^{+}, \mathrm{p}_{x, y}{ }^{-}$and $\mathrm{p}_{z}{ }^{-}$, where the superscripts + and - represent the parities of the corresponding states. When considering the SOC effects, in stage III, the strong SOC effects drive the $\mathrm{p}_{z}{ }^{+}$and $\mathrm{p}_{x, y}{ }^{-}$states to exchange positions near the Fermi level, leading to a band inversion. Note that this mechanism is different from that of $\mathrm{ZrTe}_{5} / \mathrm{HfTe}_{5}, 1 \mathrm{~T}-\mathrm{MX}_{2}$ and $1 \mathrm{~T}^{\prime}-\mathrm{MoX}_{2}$, where the SOC effects do not cause a change in the band order, whereas only a sizable gap is opened at the Fermi level. To confirm these observations, we artificially added a scaling factor $\lambda$ in front of the SOC term in the calculations, gradually increasing the strength of the SOC effects from zero to its full value and monitoring the evolution of the band structure. Taking BiPbF as an example, in Fig. 6(b) and (c), it can be observed that the direct band gap at the $\Gamma$ point decreases to zero at a critical value $\lambda=0.48$, and then it reopens again, finally reaching a value of $0.28 \mathrm{eV}$ at $\lambda=1.0$. Obviously, the topological phase transition in the current system is driven by the strength of the SOC effects, which is in agreement with the abovementioned band order analysis.

Another prominent feature of these films is that if the SOC effects are included, the energy bands at the Fermi level are spin-split, which is a signature of Rashba spin splitting (RSS). Fig. 7 presents the spin texture for the highest valence bands of all BiPbX films, where, indeed, the spin components projected to the in-plane polarization are normal to $k$ in the Brillouin zone. The RSS is characterized by the Rashba energy $E_{\mathrm{R}}$ and the corresponding momentum offset $\Delta k_{\mathrm{R}}$ away from the crossing point, which is illustrated in the inset of Fig. 7(a). From an estimation of $\Delta k_{\mathrm{R}}$ and a fitting value of the effective mass, we found that the effective Rashba constant $\alpha_{\mathrm{R}}=\hbar^{2} \Delta K / m^{*}$ reaches
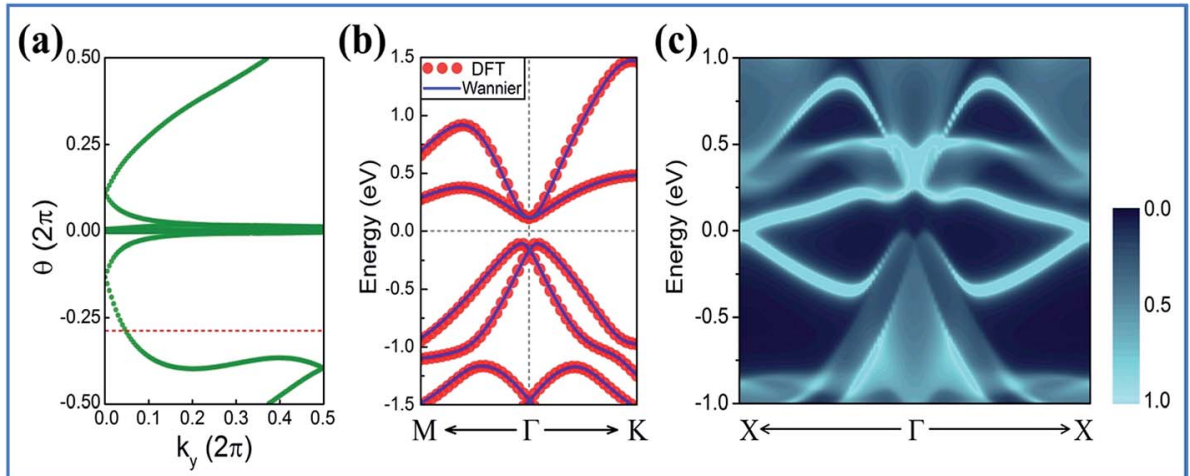

Fig. 5 (a) Evolution of the Wannier centers along $k_{y}$. The evolution lines cross the arbitrary reference line (red dashed line parallel to $k_{y}$ ) an odd number of times. (b) Comparison of the band structures obtained by DFT (red circles) and the Wannier function (blue lines). (c) Topological edge states of the $\mathrm{BiPbCl}$ monolayer with $\mathrm{SOC}$ effects, as obtained by the ab initio TB Wannier function. 


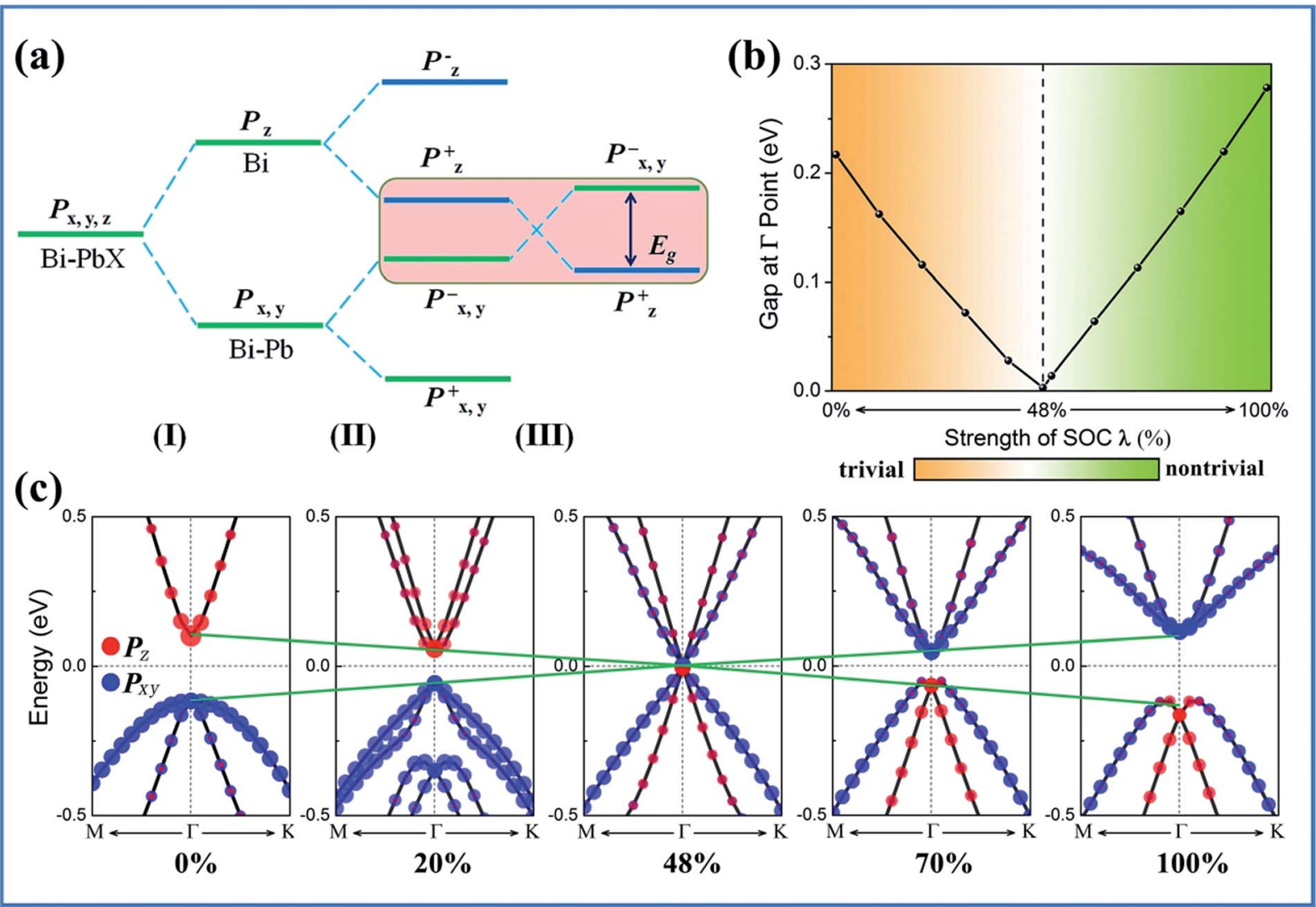

Fig. 6 (a) Schematic of the evolution from atomic Bi $\mathrm{p}_{z}$ orbitals and $\mathrm{Bi}-\mathrm{Pb} \mathrm{p}_{x, y}$ orbitals into the conduction and valence bands at the $I$ point. (b) Changes in the band gap at the $\Gamma$ point of the BiPbF monolayer as a function of SOC strength. (c) Band structures with respect to the SOC strength with $\lambda=0 \%, 20 \%, 48 \%, 70 \%$, and $100 \%$.
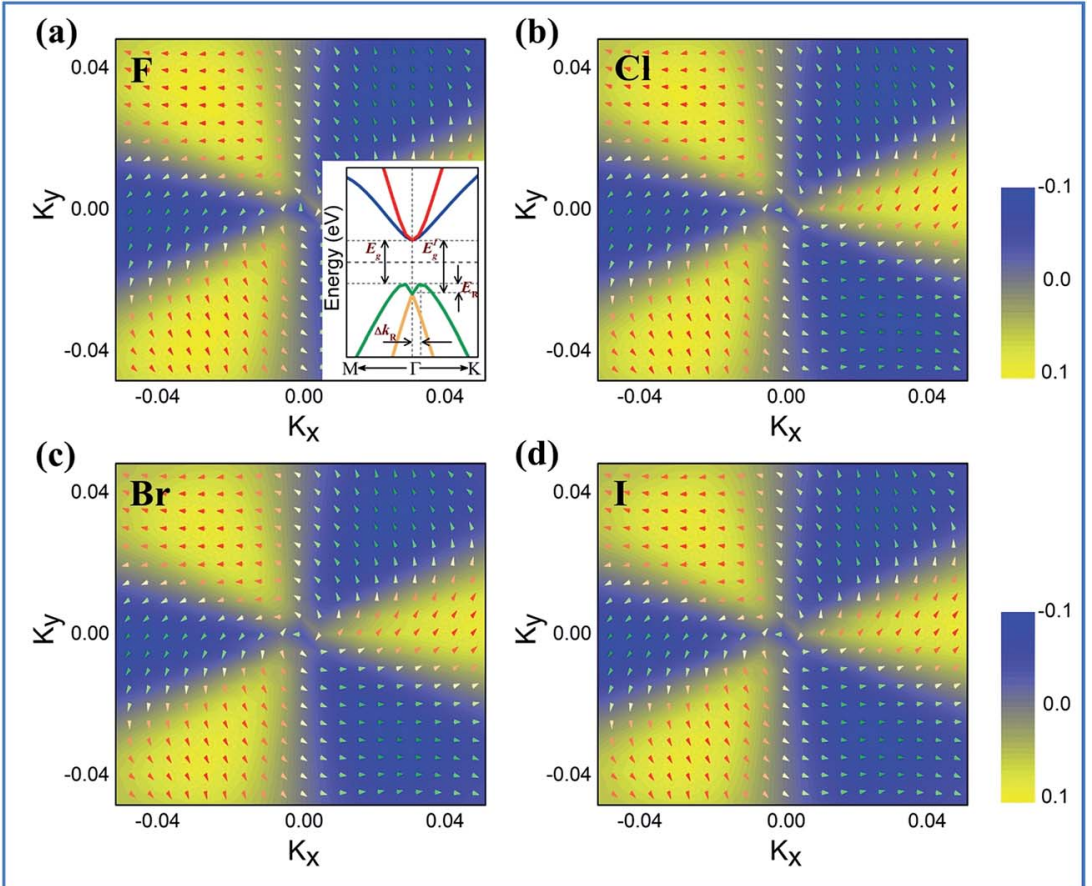

Fig. 7 Spin texture in the highest valence bands for (a) BiPbF, (b) $\mathrm{BiPbCl}$, (c) $\mathrm{BiPbBr}$, and (d) BiPbl monolayer. Arrows refer to the in-plane orientation of the spins, and the background color denotes the $z$ component of the spins. The inset in (a) is the enlarged view of the bands around the Fermi level at the $I$ point for the BiPbF structure. 


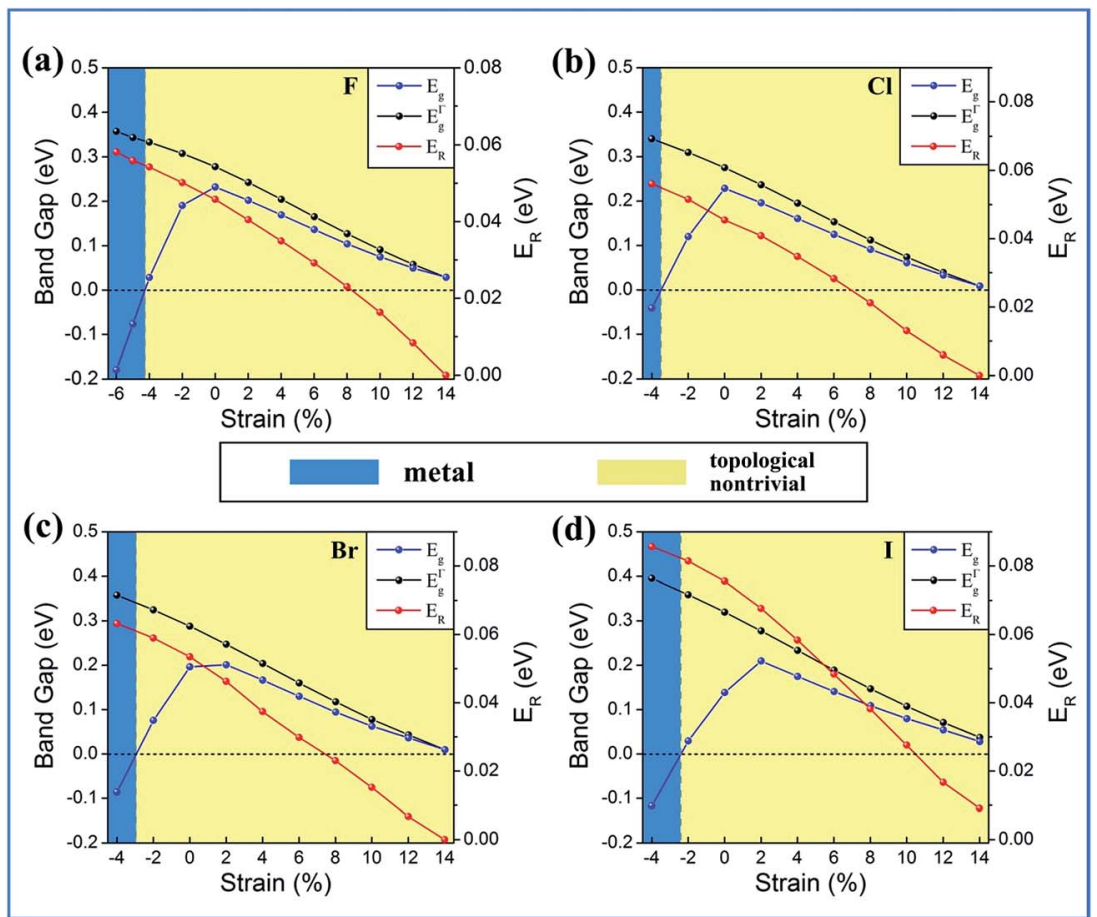

Fig. 8 Variations of the global band gap $\left(E_{\mathrm{g}}\right)$, band gap at the $\Gamma$ point $\left(E_{\mathrm{g}}{ }^{\Gamma}\right)$ and $\mathrm{RSS}\left(E_{\mathrm{R}}\right)$ as a function of external strain for $(\mathrm{a}) \mathrm{BiPbF},(\mathrm{b}) \mathrm{BiPbCl}$, (c) $\mathrm{BiPbBr}$, (d) BiPbl monolayers.

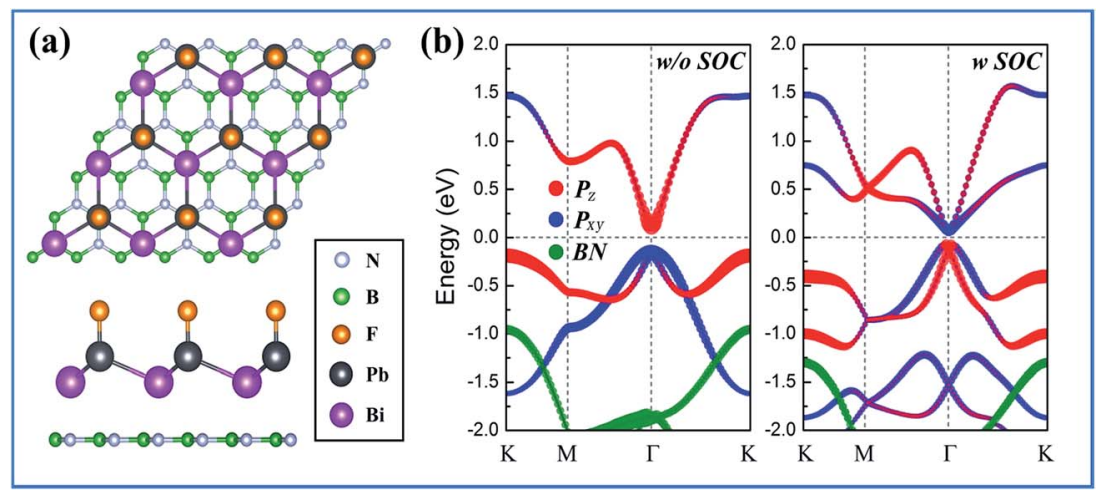

Fig. 9 (a) Schematic with a top and side view of the epitaxially grown BiPbF layer on a h-BN (0001) substrate and (b) band structures without SOC and with SOC effects for the BiPbF/h-BN (0001) HTS.

a value as large as $0.29 \mathrm{eV} \AA^{-1}$, larger than the results obtained for InGaAs/GaAs quantum dots $\left(\alpha_{\mathrm{R}} \sim 0.08-0.12 \mathrm{eV} \AA^{-1}\right)^{59}$ or InGaAs/InAlAs quantum wells $\left(\alpha_{\mathrm{R}}=0.07 \mathrm{eV} \AA^{-1}\right) .{ }^{60}$ The larger RSS in the electronic bands obtained herein provides a platform for spintronic device applications without applying a magnetic field, for instance, in spin field-effect transistors (FET).

Strain engineering is an efficient way of modulating the electronic and topological properties of 2D films. A biaxial strain can be applied by adjusting the in-plane lattice parameters, i.e., $\varepsilon=\left(a-a_{0}\right) / a_{0}$, where $a$ and $a_{0}$ are the strained and unstrained lattice constants, respectively. The calculated results are illustrated in Fig. 8. The large bulk band gap and sizable Rashba splitting coexist in all the cases. Quite interestingly, the Rashba splitting is tunable over a wide range of strains (-2-
$14 \%$ ), with the maximum bulk band gap being enhanced to 81 $\mathrm{meV}$, whereas the bulk band gap remains large enough (0.04$0.36 \mathrm{eV})$.

Finally, as illustrated in Fig. 9(a), we constructed a $\mathrm{BiPbH} / \mathrm{h}$ $\mathrm{BN}$ heterostructure (HTS) using BN as a substrate, where the lattice of h-BN matches well with the lattice of the BiPbF monolayer. After full relaxation with van der Waals (vdW) forces, ${ }^{61}$ the distance $(d)$ between adjacent layers is $4.1 \AA$, with the binding energy being $-75 \mathrm{meV}$, suggesting a typical vdW HTS. Fig. 9(b) presents the band structures with and without SOC effects. It can be observed that a few bands appear within the bulk band gap of the h-BN substrate around the Fermi level, which is mainly contributed by orbitals from the BiPbF films, according to the wave-function analysis. Upon projecting Bloch 
wave functions onto the atomic orbitals, the $\mathrm{p}_{x, y}-\mathrm{p}_{z}$ band order inversion occurs. Therefore, the BiPbX monolayer supported on the h-BN substrate is a robust TI.

In summary, we proposed a new type of $2 \mathrm{D}$ TIs based on the films consisting of group IV-V-VI elements, which exhibit a giant Rashba spin splitting, due to their peculiar polar atomic structure. The coexistence of the topological band gap and Rashba splitting is stable over a wide range of strain $(-2-14 \%)$, with even the maximum bulk band gap being enhanced to $0.36 \mathrm{eV}$, which is important for room-temperature realization of QSH states. Considering the robustness of the topologically nontrivial band structure with a h-BN substrate, this study opens a route for the design of topological spintronic devices based on the films consisting of 2D group IV-V-VI elements.

\section{Acknowledgements}

This work was supported by the National Natural Science Foundation of China (Grant No. 11434006, 61172028, and 11304121).

\section{References}

1 S. Datta and B. Das, Appl. Phys. Lett., 1990, 56, 665-667.

2 P. Gambardella and I. M. Miron, Philos. Trans. R. Soc., A, 2011, 369, 3175-3197.

3 Y. A. Bychkov and E. I. Rashba, J. Phys. C: Solid State Phys., 1984, 17, 6039-6045.

4 D. Awschalom and N. Samarth, Physics, 2009, 2, 50.

5 C. Tahan and R. Joynt, Phys. Rev. B: Condens. Matter Mater. Phys., 2005, 71, 075315.

6 D. Popović, F. Reinert, S. Hüfner, V. G. Grigoryan, M. Springborg, H. Cercellier, Y. Fagot-Revurat, B. Kierren and D. Malterre, Phys. Rev. B: Condens. Matter Mater. Phys., 2005, 72, 045419.

7 A. Varykhalov, D. Marchenko, M. R. Scholz, E. D. L. Rienks, T. K. Kim, G. Bihlmayer, J. Sánchez-Barriga and O. Rader, Phys. Rev. Lett., 2012, 108, 066804.

8 Y. M. Koroteev, G. Bihlmayer, J. E. Gayone, E. V. Chulkov, S. Blügel, P. M. Echenique and P. Hofmann, Phys. Rev. Lett., 2004, 93, 046403.

9 K. Sugawara, T. Sato, S. Souma, T. Takahashi, M. Arai and T. Sasaki, Phys. Rev. Lett., 2006, 96, 046411.

10 C. R. Ast, J. Henk, A. Ernst, L. Moreschini, M. C. Falub, D. Pacilé, P. Bruno, K. Kern and M. Grioni, Phys. Rev. Lett., 2007, 98, 186807.

11 S. Mathias, A. Ruffing, F. Deicke, M. Wiesenmayer, I. Sakar, G. Bihlmayer, E. V. Chulkov, Y. M. Koroteev, P. M. Echenique and M. Bauer, Phys. Rev. Lett., 2010, 104, 066802.

12 K. Ishizaka, M. S. Bahramy, H. Murakawa, M. Sakano, T. Shimojima, T. Sonobe, K. Koizumi, S. Shin, H. Miyahara and A. Kimura, Nat. Mater., 2011, 10, 521-526.

13 D. Di Sante, P. Barone, R. Bertacco and S. Picozzi, Adv. Mater., 2013, 25, 509-513.

14 M. Kepenekian, R. Robles, C. Katan, D. Sapori, L. Pedesseau and J. Even, ACS Nano, 2015, 9, 11557-11567.

15 Q. H. Liu, X. W. Zhang, L. B. Abdalla and A. Zunger, Adv. Funct. Mater., 2016, 26, 3259-3267.
16 J. E. Moore, Nature, 2010, 464, 194-198.

17 M. Z. Hasan and C. L. Kane, Rev. Mod. Phys., 2010, 82, 30453067.

18 X. L. Qi and S. C. Zhang, Rev. Mod. Phys., 2011, 83, 10571110.

19 H. J. Zhang, C. X. Liu, X. L. Qi, X. Dai, Z. Fang and S. C. Zhang, Nat. Phys., 2009, 5, 438-442.

20 Y. L. Chen, J. G. Analytis, J. H. Chu, Z. K. Liu, S. K. Mo, X. L. Qi, H. J. Zhang, D. H. Lu, X. Dai, Z. Fang, S. C. Zhang, I. R. Fisher, Z. Hussain and Z. X. Shen, Science, 2009, 325, 178-181.

21 Y. Xia, D. Qian, D. Hsieh, L. Wray, A. Pal, H. Lin, A. Bansil, D. Grauer, Y. S. Hor, R. J. Cava and M. Z. Hasan, Nat. Phys., 2009, 5, 398-402.

22 T. Zhang, P. Cheng, X. Chen, J. F. Jia, X. Ma, K. He, L. Wang, H. Zhang, X. Dai, Z. Fang, X. Xie and Q. K. Xue, Phys. Rev. Lett., 2009, 103, 266803.

23 B. A. Bernevig, T. L. Hughes and S. C. Zhang, Science, 2006, 314, 1757.

24 M. König, S. Wiedmann, C. Brüne, A. Roth, H. Buhmann, L. W. Molenkamp, X. L. Qi and S. C. Zhang, Science, 2007, 318, 766-770.

25 A. Roth, C. Brüne, H. Buhmann, L. W. Molenkamp, J. Maciejko, X. L. Qi and S. C. Zhang, Science, 2009, 325, 294-297.

26 C. Liu, T. L. Hughes, X. L. Qi, K. Wang and S. C. Zhang, Phys. Rev. Lett., 2008, 100, 236601.

27 C. C. Liu, W. Feng and Y. Yao, Phys. Rev. Lett., 2011, 107, 076802.

28 Y. Xu, B. Yan, H. J. Zhang, J. Wang, G. Xu, P. Tang, W. Duan and S. C. Zhang, Phys. Rev. Lett., 2013, 111, 136804.

29 Y. P. Wang, C. W. Zhang, W. X. Ji, M. J. Ren, X. L. Chen, M. Yuan and P. J. Wang, J. Phys. D: Appl. Phys., 2016, 49, 055305.

30 Z. Liu, C. X. Liu, Y. S. Wu, W. H. Duan, F. Liu and J. Wu, Phys. Rev. Lett., 2011, 107, 136805.

31 F. C. Chuang, L. Z. Yao, Z. Q. Huang, Y. T. Liu, C. H. Hsu, T. Das, H. Lin and A. Bansil, Nano Lett., 2014, 14, 2505-2508.

32 W. Luo and H. J. Xiang, Nano Lett., 2015, 15, 3230-3235.

33 J. J. Zhou, W. Feng, C. C. Liu, S. Guan and Y. Yao, Nano Lett., 2014, 14, 4767-4771.

34 W. W. Zhao, X. Chen, L. Y. Li and X. M. Zhang, Sci. Rep., $2015,5,8441$.

35 L. Y. Li, X. M. Zhang, X. Chen and M. W. Zhao, Nano Lett., 2015, 15, 1296.

36 A. Z. Wang, Z. H. Wang, A. J. Du and M. W. Zhao, Phys. Chem. Chem. Phys., 2016, 18, 22154.

37 Y. P. Wang, C. W. Zhang, W. X. Ji, P. Li, P. J. Wang and S. S. Yan, Appl. Phys. Lett., 2016, 108, 073104.

38 Y. Ma, Y. Dai, L. Kou, T. Frauenheim and T. Heine, Nano Lett., 2015, 15, 1083.

39 R. W. Zhang, C. W. Zhang, W. X. Ji, S. S. Li, S. J. Hu, S. S. Yan, P. Li, P. J. Wang and F. Li, New J. Phys., 2015, 17, 083036.

40 H. Zhao, C. W. Zhang, W. X. Ji, R. W. Zhang, S. S. Li, S. S. Yan, B. M. Zhang, P. Li and P. J. Wang, Sci. Rep., 2016, 6, 20152.

41 R. W. Zhang, C. W. Zhang, W. X. Ji, S. S. Li, S. S. Yan and P. J. Wang, Sci. Rep., 2016, 6, 21351. 
42 Y. P. Wang, W. X. Ji, C. W. Zhang, P. Li, F. Li, M. J. Ren, X. L. Chen, M. Yuan and P. J. Wang, Sci. Rep., 2016, 6, 20342.

43 Y. Z. Jia, W. X. Ji, C. W. Zhang, P. Li, M. J. Ren and P. J. Wang, J. Mater. Chem. C, 2016, 4(37), 8750-8757.

44 R. W. Zhang, C. W. Zhang, W. X. Ji, P. Li, P. J. Wang, S. S. Li and S. S. Yan, Appl. Phys. Lett., 2016, 109, 182109.

45 R. W. Zhang, C. W. Zhang, W. X. Ji, S. S. Li, S. S. Yan, S. J. Hu, P. Li and P. J. Wang, J. Mater. Chem. C, 2016, 4, 2088.

46 X. Wan, A. M. Turner, A. Vishwanath and S. Y. Savrasov, Phys. Rev. B: Condens. Matter Mater. Phys., 2011, 83, 205101.

47 J. Wang, X. Chen, B. F. Zhu and S. C. Zhang, Phys. Rev. B: Condens. Matter Mater. Phys., 2012, 85, 235131.

48 G. Kresse and J. Furthmüller, Phys. Rev. B: Condens. Matter Mater. Phys., 1996, 54, 11169-11186.

49 G. Kresse and J. Hafner, Phys. Rev. B: Condens. Matter Mater. Phys., 1994, 49, 14251-14269.

50 G. Kresse and D. Joubert, Phys. Rev. B: Condens. Matter Mater. Phys., 1999, 59, 1758-1775.

51 J. P. Perdew and Y. Wang, Phys. Rev. B: Condens. Matter Mater. Phys., 1992, 45, 13244-13249.
52 J. Heyd, G. E. Scuseria and M. Ernzerhof, J. Chem. Phys., 2006, 124, 219906.

53 J. Heyd, G. E. Scuseria and M. Ernzerhof, J. Chem. Phys., 2003, 118, 8207.

54 C. W. Zhang and S. S. Yan, J. Phys. Chem. C, 2012, 116, 41634166.

55 F. B. Zheng and C. W. Zhang, Nanoscale Res. Lett., 2012, 7, 422.

56 H. Weng, X. Dai and Z. Fang, Phys. Rev. X, 2014, 4(1), 011002.

57 M. P. Lopez Sancho, J. M. Lopez Sancho, J. M. L. Sancho and J. Rubio, J. Phys. F: Met. Phys., 1985, 15, 851.

58 R. Yu, X. L. Qi, A. Bernevig, Z. Fang and X. Dai, Phys. Rev. B: Condens. Matter Mater. Phys., 2011, 84, 075119.

59 S. M. Huang, A. O. Badrutdinov, L. Serra, T. Kodera, T. Nakaoka, N. Kumagai, Y. Arakawa, D. A. Tayurskii, K. Kono and K. Ono, Phys. Rev. B: Condens. Matter Mater. Phys., 2011, 84, 085325.

60 J. Nitta, T. Akazaki, H. Takayanagi and T. Enoki, Phys. Rev. Lett., 1997, 78, 1335-1338.

61 J. Klimeš, D. R. Bowler and A. Michaelides, Phys. Rev. B: Condens. Matter Mater. Phys., 2011, 83, 195131. 